\section{Perfil dos ensaios clínicos envolvendo crianças brasileiras}

\author{
Profile of clinical trials enrolling Brazilian children
}

\section{Perfil de los ensayos clínicos involucrando niños brasileños}

\section{Resumo}

Este estudo visou a caracterizar os ensaios clínicos com medicamentos envolvendo crianças e adolescentes brasileiros, registrados nas bases de dados do Clinical Trials e da Registro Brasileiro de Ensaios Clinicos (ReBEC), entre os anos de 1994 e 2014. Apenas 462 ensaios clinicos envolveram brasileiros nessa faixa etária. A partir de 2003, houve aumento no número de registros, com expressiva queda em 2011. Dentre esses, 35,5\% foram sediados no Brasil. Os ensaios clinicos internacionais foram majoritariamente conduzidos por empresas norte-americanas. Em ambos os casos, a indústria multinacional foi a principal fonte de apoio financeiro. Predominaram ensaios clinicos de fase III com antivirais em formas farmacêuticas injetáveis e sólidas orais. Os ensaios clinicos nacionais apresentaram maior variação quanto às formas farmacêuticas e maior porcentual de formulações líquidas investigadas, em comparação aos internacionais. Além da forte dependência externa para a realização dos ensaios clínicos, destacou-se o desafio para o cuidado pediátrico no Brasil, que apresenta peculiaridades epidemiológicas em um ambiente propício ao uso de medicamentos não licenciados para crianças.

Ensaio Clínico; Pediatria; Sistemas de Informação em Saúde
Jean Mendes de Lucena Vieira 1

Elisangela da Costa Lima 1

Marcelo Gerardin Poirot Land 2

Miriam Ventura 3

Helena Lutescia Luna Coelho 4

doi: 10.1590/0102-311X00169515

\section{Correspondência}

J. M. L. Vieira

Observatório de Vigilância e Uso de Medicamentos, Faculdade de Farmácia, Universidade Federal do Rio de Janeiro. Av. Carlos Chagas Filho 373, Cidade Universitária, Rio de Janeiro, RJ 21944-970, Brasil.

jeanmlvieira@hotmail.com

1 Faculdade de Farmácia, Universidade Federal do Rio de Janeiro, Rio de Janeiro, Brasil.

2 Instituto de Pediatria e Puericultura Martagão Gesteira,

Universidade Federal do Rio de Janeiro, Rio de Janeiro, Brasil.

3 Instituto de Estudos em Saúde Coletiva, Universidade Federal do Rio de Janeiro, Rio de Janeiro, Brasil.

4 Programa de Pós-graduação em Ciências Farmacêuticas,

Universidade Federal do Ceará, Fortaleza, Brasil. 


\section{Introdução}

A utilização de medicamentos seguros e eficazes em pediatria ainda constitui um problema, ocasionado pela escassez de formulações adequadas e de estudos clínicos e farmacoepidemiológicos que contemplem esta população 1,2,3. Em suas diversas faixas etárias, crianças e adolescentes são expostos a medicamentos cujo benefício clínico só é conhecido em adultos. Nessa condição, o risco da farmacoterapia é maior, sobretudo pelas diferenças no processo fisiopatológico e nos aspectos farmacocinéticos e farmacodinâmicos de fármacos e excipientes no organismo em constante desenvolvimento e em diferentes níveis de maturação, não necessariamente graduais ou previsíveis 4,5.

Um ensaio clínico pode ser definido como um estudo de intervenção em seres humanos com a finalidade de descobrir ou confirmar os efeitos clínicos, farmacológicos e/ou farmacodinâmicos dos produtos sob investigação, bem como avaliar o perfil de segurança. Assim, os ensaios clínicos subsidiam o registro do produto ou sua alteração junto à agência reguladora sanitária 6 .

No que tange às formulações pediátricas, (i) o número restrito de sujeitos disponíveis para a participação em pesquisas, (ii) seus elevados custos marginais, (iii) o reduzido mercado e (iv) a complexidade ética na análise dos estudos são apontados como os principais fatores que desestimulam a indústria farmacêutica a desenvolver medicamentos para crianças em todo o mundo 7 . Consequentemente, em condições clínicas graves - ou mesmo em situações frequentes - são utilizados medicamentos sem evidências geradas em estudos controlados realizados para as diversas faixas etárias da população pediátrica 8 .

Iniciativas regulatórias ocorridas, principalmente nos Estados Unidos e na União Europeia, a partir de 1997, incluíram políticas fortes de estímulo à pesquisa e ao desenvolvimento de formulações pediátricas. Essas ações vêm impactando positivamente o registro de ensaio clínico em crianças e neonatos nas bases internacionais ${ }^{9}$. No Brasil, desde o ano de 1988, a realização de pesquisas em seres humanos, incluindo os ensaios clínicos, está sujeita à regulamentação do Conselho Nacional de Saúde (CNS) e da Agência Nacional de Vigilância Sanitária (Anvisa) 10. A principal norma regulamentadora é a Resolução CNS no 466/2012, que estabelece o sistema nacional de análise ética, Comitê de Ética em Pesquisa, Comissão Nacional de Ética em Pesquisa (CEP-CONEP), as normas e o fluxo de tramitação dos protocolos de pesquisa 11 .

A publicação de novas regulamentações para pesquisas em âmbitos nacional e internacional, a oferta de modelos de financiamento científico e tecnológico, bem como o estímulo às parcerias entre universidades e empresas, em muito contribuíram para o amadurecimento e consolidação da pesquisa clínica no Brasil 12. No entanto, a análise ética de ensaios clínicos pediátricos é, relativamente, mais complexa 13. Apesar de qualquer estudo clínico apresentar riscos e benefícios, o envolvimento de crianças deve considerar outras implicações, tais como o desconforto, a dor, o medo, a ausência dos pais, os efeitos sobre o crescimento ou sobre o desenvolvimento de órgãos, além das limitações na obtenção de amostras 14 . Temores relacionados à eficácia do tratamento, aos eventos adversos conhecidos e desconhecidos, à possível menor prioridade dos interesses da criança versus aos dos investigadores são as principais barreiras para que os pais autorizem a participação dos filhos 14 .

A ausência de profissionais com expertise no campo também acentua o problema. A proporção de médicos brasileiros com experiência em pesquisa clínica é pequena, sobretudo com crianças. Além disso, a área de pediatria no país viveu, até recentemente, uma situação de carência de profissionais, e unidades de saúde pediátricas foram fechadas 15 , processo que está revertendo atualmente.

Considerando o cenário apresentado, o presente trabalho objetivou caracterizar os ensaios clínicos de medicamentos envolvendo crianças e adolescentes brasileiros, registrados em bases de dados públicas, buscando analisar a situação brasileira.

\section{Metodologia}

Foi realizado um estudo exploratório retrospectivo em duas bases de dados informatizadas para registros de ensaios clínicos: (i) Clinical Trials, criado em 2000; e (ii) Registro Brasileiro de Ensaios Clínicos (ReBEC), criado em 2010. Esses sistemas constituem as maiores e principais bases de livre acesso e registro voluntário de ensaios clínicos internacionais e nacionais, respectivamente 16,17. 
Além da quantificação do volume de estudos por ano, foi possível selecionar os ensaios clínicos: (i) caracterizados como estudos de intervenção; (ii) registrados até o ano de 2014; (iii) que envolveram participantes brasileiros; (iv) com idades entre 0 e 17 anos, utilizando o recurso de busca avançada em ambas as bases. Foram excluídos estudos em duplicatas, bem como os que não envolveram participantes brasileiros.

Coletamos as seguintes informações: ano de início do ensaio clínico, fase clínica, instituição ou empresa patrocinadora, país sede do patrocinador, região e estado brasileiro de realização, fonte financeira de apoio, condição/problema de saúde tratado, medicamento investigado e forma farmacêutica.

A classificação da condição de saúde investigada considerou a determinação da Classificação Internacional de Doenças, 10a revisão (CID-10). Os medicamentos investigados nos ensaios clínicos (intervenções) foram classificados de acordo com o Anatomical Therapeutic Chemical Code (ATC) 18.

Visando a cotejar as intervenções investigadas com o perfil brasileiro de morbimortalidade infantil, foram utilizadas informações disponibilizadas no banco de dados do Departamento de Informática do Sistema Único de Saúde (DATASUS), referentes ao ano de 2012.

Os dados foram organizados em planilha do programa Microsoft Excel 2013 (Microsoft Corp., Estados Unidos). O programa IBM SPSS, versão 22 (IBM Corp., Armonk, Estados Unidos) foi empregado para análise da distribuição de frequências e comparação das variáveis de interesse coletadas.

\section{Resultados}

\section{Caracterização dos ensaios clínicos}

O registro de ensaios clínicos envolvendo crianças cadastradas nas bases de dados analisadas aumentou 23 vezes ao longo do período estudado. Contudo, notou-se que no período entre 2011 e 2013 o número de ensaios clínicos patrocinados por multinacionais caiu 16\% e a quantidade total de ensaios clínicos envolvendo crianças no mundo cresceu $3 \%$.

A quantidade de ensaios clínicos pediátricos realizados no Brasil também sofreu uma queda marcante entre 2011 e 2014, ainda não recuperada, após um longo período de crescimento (Figura 1).

\section{Figura 1}

Distribuição gráfica dos ensaios clínicos envolvendo crianças segundo o ano de início registrado (1994-2014).

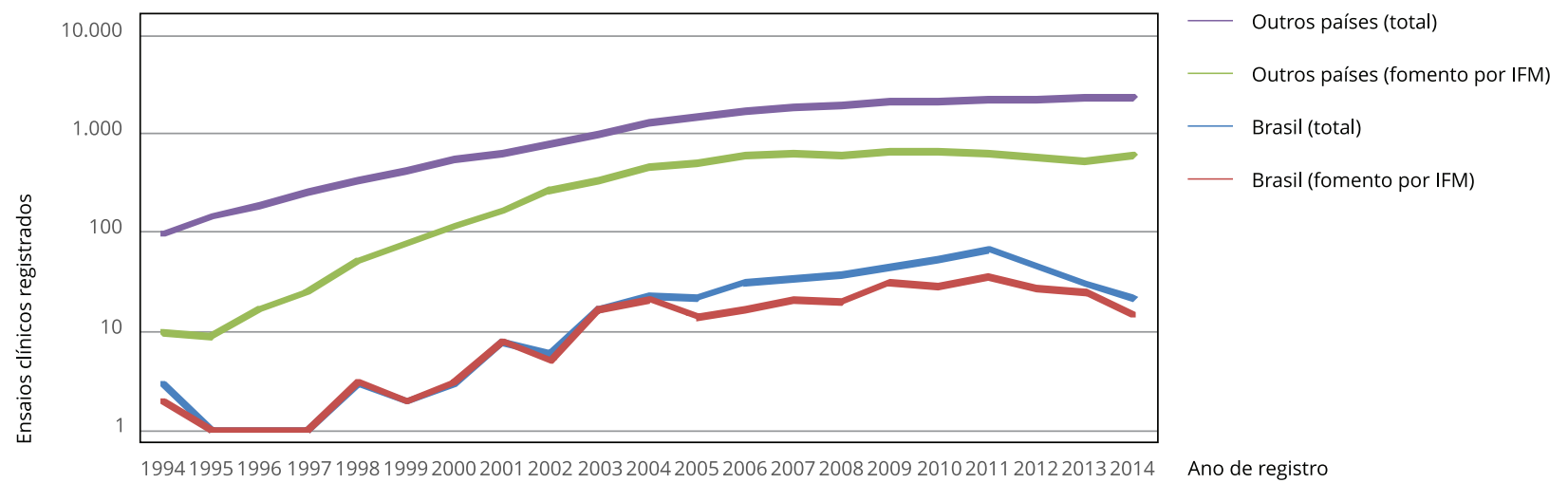

Nota: formato por IFM: quantidade referente aos ensaios clínicos registrados que foram patrocinados por Indústrias Farmacêuticas Multinacionais. Escala logarítmica.

Fonte: Clinical Trials e Registro Brasileiro de Ensaios Clínicos (1994-2014). 
O uso da ferramenta de busca avançada para a seleção dos ensaios clínicos que envolveram crianças brasileiras permitiu a identificação de nove registros no ReBEC e 453 no Clinical Trials, após aplicação dos critérios de inclusão e exclusão. Esses 462 ensaios clínicos corresponderam a 0,24\% do volume total de 187.213 registrados em ambas as bases entre 1994 e 2014.

O Brasil esteve definido como sede (coordenador do estudo) em 35,5\% dos ensaios clínicos registrados - denominados como nacionais nesta pesquisa -, seguido pelos Estados Unidos (27\%) e Suíça $(9,7 \%)$ (Tabela 1). Em média, as pesquisas foram conduzidas em sete países, com mediana igual a quatro.

O número de participantes por estudo variou entre cinco (estudo registrado pela Universidade Federal de São Paulo sobre gamaglobulina) e 63.227 (estudo da GlaxoSmithKline sobre vacina contra rotavírus). A moda e a mediana de participantes foram de 40 e 210, respectivamente. Quanto ao local de residência das crianças participantes da pesquisa, observou-se que além de brasileiros foram incluídos participantes de outros 98 países. A maioria dos estudos nacionais não incluiu participantes de outras nacionalidades.

Tabela 1

Distribuição dos ensaios clínicos pediátricos quanto ao país de origem, fase clínica, coordenador e fonte de apoio financeiro. Clinical Trials e ReBEC, 1994-2014 ( $\mathrm{N}=462)$.

\begin{tabular}{|c|c|c|c|c|c|c|}
\hline Caracterização quanto à origem & Nacional & $\mathbf{n}$ & $\%$ & Internacional & $\mathbf{n}$ & $\%$ \\
\hline \multirow[t]{10}{*}{ País sede } & Brasil & 164 & 100 & Estados Unidos & 124 & 42 \\
\hline & & & & Suíça & 45 & 15 \\
\hline & & & & Inglaterra & 33 & 11 \\
\hline & & & & França & 24 & 8 \\
\hline & & & & Bélgica & 18 & 6 \\
\hline & & & & Japão & 17 & 6 \\
\hline & & & & Alemanha & 15 & 5 \\
\hline & & & & Dinamarca & 13 & 4 \\
\hline & & & & Irlanda & 3 & 1 \\
\hline & & & & $\begin{array}{c}\text { Outros (Portugal, } \\
\text { Bangladesh, Canadá e } \\
\text { Holanda) }\end{array}$ & 6 & 2 \\
\hline \multirow[t]{4}{*}{ Fase clínica } & 1 & 5 & 3 & 1 & 24 & 8 \\
\hline & II & 54 & 30 & II & 60 & 19 \\
\hline & III & 76 & 44 & III & 195 & 61 \\
\hline & IV & 47 & 23 & IV & 39 & 12 \\
\hline \multirow[t]{2}{*}{$\begin{array}{l}\text { Instituição/Empresa coordenadora do } \\
\text { estudo }\end{array}$} & $\begin{array}{l}\text { Instituições de pesquisa, } \\
\text { empresas públicas ou } \\
\text { universidades }\end{array}$ & 138 & 84 & $\begin{array}{l}\text { Instituições de pesquisa, } \\
\text { universidades, consórcios e } \\
\text { grupos de pesquisa }\end{array}$ & 33 & 11 \\
\hline & $\begin{array}{c}\text { Empresas farmacêuticas } \\
\text { nacionais }\end{array}$ & 26 & 16 & $\begin{array}{c}\text { Empresas farmacêuticas } \\
\text { multinacionais }\end{array}$ & 265 & 89 \\
\hline \multirow[t]{4}{*}{ Fonte de apoio financeiro } & CNPq & 12 & 7 & $\begin{array}{c}\text { Coordenador do ensaio } \\
\text { clínico }\end{array}$ & 298 & 100 \\
\hline & $\begin{array}{c}\text { Fundações Estaduais de } \\
\text { Pesquisa }\end{array}$ & 22 & 13 & & & \\
\hline & $\begin{array}{c}\text { Empresa farmacêutica } \\
\text { multinacional }\end{array}$ & 104 & 64 & & & \\
\hline & Coordenador do ensaio clínico & 26 & 16 & & & \\
\hline Total & & 164 & 100 & & 298 & 100 \\
\hline
\end{tabular}

CNPq: Conselho Nacional de Desenvolvimento Científico e Tecnológico; ReBEC: Registro Brasileiro de Ensaios Clínicos.

Nota: alguns estudos envolveram mais de uma fase clínica. 
Empresas multinacionais farmacêuticas, sediadas na América do Norte (51\%) e Europa (29\%), fomentaram a maior parte dos estudos registrados, com destaque para Novartis, Sanofi, Glaxo SmithKline e Bristol-Myers Squibb que patrocinaram 88 ensaios clínicos. Apenas em $8 \%$ dos ensaios clínicos foram declarados apoio financeiro do governo brasileiro (pelo Conselho Nacional de Desenvolvimento Científico e Tecnológico e/ou Fundações Estaduais de Amparo à Pesquisa).

Em relação à fase clínica, houve predomínio dos estudos de fase III tanto para ensaios realizados com patrocínio nacional $(\mathrm{n}=76 ; 44 \%)$ como para os patrocinados por multinacionais $(\mathrm{n}=195 ; 61 \%)$. Além disso, destaca-se um maior número de estudos de fase IV com origem e patrocínio nacional $(\mathrm{n}=47 ; 23 \%)$ do que com origem e patrocínio estrangeiros ( $\mathrm{n}=39 ; 12 \%)$ (Tabela 1$)$.

Os ensaios clínicos brasileiros foram realizados por instituições localizadas nas regiões Sudeste (64,5\%), Sul (14\%), Nordeste (14,5\%) e Centro-oeste (7\%). O Estado de São Paulo concentrou 43\% dos ensaios clínicos. Não foram identificados ensaios clínicos registrados por instituições/empresas localizadas na Região Norte do país.

\section{Intervenções investigadas}

Formas farmacêuticas injetáveis e sólidas orais predominaram nos ensaios clínicos registrados. Contudo, os estudos nacionais investigaram um percentual maior de medicamentos em formulações líquidas orais e apresentaram maior variação quanto às vias de administração investigadas, em comparação aos internacionais (Tabela 2).

Os ensaios clínicos nacionais contemplaram medicamentos com ação no sistema nervoso central (17\%), digestivo (12\%), respiratório (10\%) e na pele (10\%). Dentre os ensaios clínicos internacionais, quase a metade investigou medicamentos anti-infecciosos de uso sistêmico (30\%) e antineoplásicos (18\%), seguidos pelos medicamentos com ação digestiva (10\%) e hematológica (10\%) (Tabela 3).

No grupo J, os antivirais - especialmente aqueles indicados para o tratamento de infecções pelo vírus da imunodeficiência humana como adefovir, darunavir, ritonavir, estavudina, tenofovir, lami-

Tabela 2

Distribuição dos ensaios clínicos segundo as formas farmacêuticas investigadas, origem e idade dos sujeitos. Clinical Trials e ReBEC, $1994-2014$.

\begin{tabular}{|c|c|c|c|c|c|c|c|c|c|c|}
\hline \multirow[t]{3}{*}{ Origem/Idade mínima (anos) } & \multicolumn{8}{|c|}{ Forma farmacêutica } & \multicolumn{2}{|c|}{ Total } \\
\hline & \multicolumn{2}{|c|}{ Sólido oral } & \multicolumn{2}{|c|}{ Injetável } & \multicolumn{2}{|c|}{ Líquido oral } & \multicolumn{2}{|c|}{ Outros * } & \multirow[b]{2}{*}{$n$} & \multirow[b]{2}{*}{$\%$} \\
\hline & $\mathbf{n}$ & $\%$ & $\mathbf{n}$ & $\%$ & $\mathbf{n}$ & $\%$ & $\mathbf{n}$ & $\%$ & & \\
\hline Nacionais & 48 & 28 & 53 & 32 & 32 & 19 & 35 & 21 & 168 & 100 \\
\hline $0-1$ & 13 & 34 & 15 & 40 & 8 & 21 & 2 & 5 & 38 & 7 \\
\hline$\geq 1-4$ & 17 & 28 & 25 & 41 & 11 & 18 & 8 & 13 & 61 & 12 \\
\hline$\geq 4-6$ & 15 & 26 & 24 & 41 & 9 & 16 & 10 & 17 & 58 & 11 \\
\hline$\geq 6-10$ & 23 & 31 & 25 & 35 & 12 & 17 & 12 & 17 & 72 & 13 \\
\hline$\geq 10-12$ & 25 & 35 & 25 & 35 & 10 & 14 & 12 & 16 & 72 & 13 \\
\hline$\geq 12-16$ & 30 & 26 & 39 & 35 & 19 & 16 & 27 & 23 & 115 & 22 \\
\hline$\geq 16-17$ & 29 & 27 & 37 & 35 & 16 & 15 & 24 & 23 & 106 & 22 \\
\hline Internacionais & 130 & 41 & 132 & 42 & 35 & 11 & 18 & 6 & 315 & 100 \\
\hline $0-1$ & 27 & 34 & 35 & 45 & 13 & 17 & 3 & 4 & 78 & 7 \\
\hline$\geq 1-4$ & 46 & 37 & 53 & 43 & 21 & 17 & 4 & 3 & 124 & 12 \\
\hline$\geq 4-6$ & 48 & 36 & 59 & 44 & 22 & 16 & 5 & 4 & 134 & 13 \\
\hline$\geq 6-10$ & 46 & 34 & 62 & 47 & 19 & 14 & 7 & 5 & 134 & 13 \\
\hline$\geq 10-12$ & 54 & 36 & 63 & 43 & 20 & 14 & 10 & 7 & 147 & 14 \\
\hline$\geq 12-16$ & 68 & 34 & 91 & 47 & 24 & 12 & 14 & 7 & 197 & 19 \\
\hline$\geq 16-17$ & 94 & 42 & 93 & 41 & 23 & 10 & 15 & 7 & 225 & 22 \\
\hline
\end{tabular}

ReBEC: Registro Brasileiro de Ensaios Clínicos.

Nota: alguns estudos investigaram mais de uma forma farmacêutica.

* Formas farmacêuticas semissólidas e gasosas. 
Tabela 3

Classes de medicamentos envolvidas nos estudos clínicos pediátricos. Clinical Trials e ReBEC, 1994-2014.

\begin{tabular}{|c|c|c|c|c|c|}
\hline \multirow[t]{2}{*}{ Classificação dos medicamentos (Sistema ATC) } & \multicolumn{2}{|c|}{ Nacional } & \multicolumn{2}{|c|}{ Internacional } & \multirow[t]{2}{*}{ Total } \\
\hline & $\mathbf{n}$ & $\%$ & $\mathbf{n}$ & $\%$ & \\
\hline A. Medicamentos com ação digestiva e para o metabolismo & 22 & 13 & 31 & 10 & 53 \\
\hline B. Medicamentos com ação no sangue e órgãos hematopoiéticos & 10 & 6 & 30 & 10 & 40 \\
\hline C. Medicamentos com ação cardiovascular & 13 & 8 & 12 & 4 & 25 \\
\hline D. Medicamentos para uso dermatológico & 16 & 10 & 2 & 1 & 18 \\
\hline G. Medicamentos com ação no aparelho geniturinário & 6 & 4 & 13 & 4 & 19 \\
\hline H. Preparações hormonais sistêmicas & 5 & 3 & 0 & 0 & 5 \\
\hline J. Anti-infecciosos para uso sistêmico & 14 & 8 & 88 & 30 & 102 \\
\hline L. Agentes antineoplásicos e imunomoduladores & 14 & 8 & 55 & 18 & 69 \\
\hline M. Medicamentos com ação no sistema musculoesquelético & 6 & 4 & 11 & 4 & 17 \\
\hline N. Medicamentos com ação no sistema nervoso & 28 & 17 & 14 & 5 & 42 \\
\hline P. Medicamentos antiparasitários & 5 & 3 & 8 & 3 & 13 \\
\hline R. Medicamentos com ação no sistema respiratório & 17 & 10 & 22 & 7 & 39 \\
\hline S. Produtos para uso oftalmológico ou otológico & 6 & 4 & 3 & 1 & 9 \\
\hline V. Outros produtos terapêuticos, incluindo radiofármacos & 4 & 2 & 8 & 3 & 12 \\
\hline Não classificado (informação confidencial) & 0 & 0 & 1 & 0 & 1 \\
\hline Total * & 166 & 100 & 298 & 100 & 465 \\
\hline
\end{tabular}

* Alguns estudos investigaram mais de uma classe de medicamentos.

ATC: Anatomical Therapeutic Chemical Code; ReBEC: Registro Brasileiro de Ensaios Clínicos.

vudina, lopinavir, zidovudina, nevirapina, nelfinavir, incluindo associações - foram analisados em um número maior de estudos (22\%), comparados aos antibacterianos (7\%). Dentre o conjunto de antineoplásicos (15\%) pesquisados, destacaram-se os indicados para o tratamento da leucemia (dasatinibe, nilotinibe, imatinibe e bendamustina). Formoterol, budesonida, terbutalina, ciclesonida, salbutamol e anticorpos monoclonais (lebrikizumab, reslizumab e omalizumab) também se destacaram no grupo de antiasmáticos investigados (7\%).

\section{Discussão}

O evidente crescimento do número de registros dos ensaios clínicos pediátricos, a partir de 2003, é apontado como um reflexo da publicação do Best Pharmaceuticals for Children Act (BPCA) pela agência reguladora estadunidense, que ofereceu período adicional de exclusividade da patente de medicamentos testados em crianças em 2002 19. Segundo Hoppu et al. 20, estratégias nos Estados Unidos e na União Europeia disponibilizaram informações importantes para a terapia pediátrica, apesar dos ensaios clínicos não estarem totalmente alinhados às necessidades de saúde prioritárias em crianças brasileiras.

Entende-se que é crucial o interesse público e político sobre medicamentos pediátricos para sucesso dessas iniciativas em longo prazo 20 . No entanto, a estagnação e a queda - no caso dos ensaios clínicos patrocinados por multinacionais - observadas são preocupantes. Vickers 21 aponta a existência de um consenso sobre a "quebra" do sistema de ensaios clínicos envolvendo adultos e crianças nos Estados Unidos, com aumento nos gastos e menor inovação. A necessidade de maior investimento associada à crise econômica mundial vem impactando a pesquisa e desenvolvimento (P\&D) de medicamentos 22 . Pamolli et al. 23 apontaram que os esforços internacionais em P\&D vêm sendo focados em projetos com altos valores nas vendas como medicamentos para o tratamento de câncer, obesidade, infecções, artrite reumatoide e diabetes, como observado no presente trabalho. A taxa de crescimento dos gastos em P\&D nos Estados Unidos foi de 6,3\% entre 1994 e 2004, e caiu para 0,8\% entre 2004 e 2012. A diminuição de investimentos privados no mesmo país também foi apontada por Moses et al. 24 . 
No Brasil, a maior parte dos estudos pediátricos recebeu financiamento de empresas farmacêuticas, tal como ocorre nos demais ensaios clínicos realizados com adultos 25 . O predomínio de estudos de fase III, em que os pesquisadores envolvidos nos vários centros apenas executam o projeto sem a participação no delineamento da pesquisa ou na futura análise e discussão dos resultados, reforça a situação de dependência produtiva e tecnológica 12,26. Observou-se, ainda, o expressivo percentual de estudos de fase IV realizados e patrocinados nacionalmente em relação aos que envolvem empresas multinacionais. Esse resultado indica maior acompanhamento e avaliação dos efeitos dos medicamentos em longo prazo sobre os desfechos clínicos humanos no Brasil 27 (Tabela 1).

As pesquisas existentes parecem estar relacionadas, portanto, aos aspectos gerais já apontados na literatura. Esses aspectos: (i) facilidade no recrutamento e acompanhamento de pacientes diversificados pelo sistema público em um contexto de insuficiente acesso ao tratamento médico; (ii) alta incidência de doenças que são prevalentes em países desenvolvidos; e (iii) normas éticas compatíveis com outros países tornam o Brasil atrativo para a terceirização de ensaios clínicos por empresas multinacionais $28,29,30$. Tais aspectos, somados aos incentivos concedidos pelas agências norte-americana e europeia citados anteriormente, parecem ter refletido no aumento do número de ensaios clínicos com crianças brasileiras, a partir de 2003, apesar da demanda de um prazo maior para aprovação ética no país quando comparado com outras regiões do mundo 25 .

Todavia, o contexto internacional foi extremamente sentido no Brasil. Segundo os registros de ensaios clínicos, a quantidade de estudos pediátricos iniciados nos anos de 2013 e 2014 foi a metade da observada em 2012, voltando ao mesmo patamar do ano de 2002 (Figura 1), quando os investimentos em pesquisa clínica no país representaram cerca de 110 milhões de reais 31 .

Tal decréscimo é inquietante, visto o extenso uso off label ou não licenciado de medicamentos de grande importância clínica para crianças em todo o mundo 3,8. A carência de formulações pediátricas adequadas no Brasil, tal como em outros contextos, é bastante relatada na literatura 1,3,4,32. O desenvolvimento de formas farmacêuticas apropriadas às crianças - líquidos orais com sabor e aroma aceitáveis, minitabletes ou minicomprimidos dispersíveis ou de fácil deglutição - ainda é um desafio, apesar da existência de capacidade técnico-científica e industrial no país 3,33. Apenas em situações agudas graves, a apresentação injetável é a de escolha, por permitir maior precisão na dose administrada às crianças. Ressalta-se que as apresentações injetáveis foram mais prevalentes nos ensaios clínicos analisados, tanto em nível nacional como internacional (Tabela 2).

Os resultados desta pesquisa não trazem nenhum alento sobre esta questão, considerando o ínfimo percentual de ensaios clínicos com soluções orais ou novas formas farmacêuticas adequadas a crianças, principalmente nos estudos patrocinados por multinacionais (11\%). Foi registrado apenas um trabalho envolvendo o uso de minicomprimidos, patrocinado pelo grupo farmacêutico suíço Novartis, que investigou o uso de aliskireno no tratamento de hipertensão em participantes de pesquisa de 6-17 anos. A transformação de formulações sólidas no momento do uso, para adequação da dose ou facilidade de ingestão pela criança, é uma prática de rotina, embora esteja associada a uma maior frequência de erros de medicação e eventos adversos ${ }^{34}$. Os aspectos relacionados ao perfil de segurança da formulação incluem, ainda, a escolha do excipiente. Estudo realizado em uma Unidade de Tratamento Intensivo (UTI) neonatal de um hospital de Brasília, identificou 57 diferentes excipientes prejudiciais ou potencialmente nocivos aos quais os recém-nascidos (98,7\%) acompanhados no estudo foram expostos 35 . No entanto, no Brasil são inexistentes ou insuficientes as iniciativas regulatórias, fomento para pesquisa clínica em pediatria e investimento para a formação de pesquisadores no campo 36 .

$\mathrm{Na}$ análise dos principais problemas de saúde em crianças, prevaleceram doenças do aparelho respiratório e as complicações relacionadas ao parto e puerpério (DATASUS. Informações de saúde: estatísticas vitais - mortalidade Brasil. http://tabnet.datasus.gov.br/cgi/tabcgi.exe?sim/cnv/obt10uf. def; Morbidade hospitalar do SUS - por local de internação. http://tabnet.datasus.gov.br/cgi/tabcgi. exe?sih/cnv/nirj.def). Doenças relacionadas à gravidez e ao parto, infecto-parasitárias e do aparelho digestório se destacaram em relação à morbidade (Figura 2). Quanto aos óbitos, excluindo-se aqueles relativos às malformações congênitas ou complicações do parto e puerpério, destacaram-se as neoplasias e os problemas relacionados aos sistemas nervoso e respiratório. Observou-se que os medicamentos investigados nos estudos registrados envolvendo crianças brasileiras não corresponderam integralmente às necessidades sanitárias pediátricas brasileiras. 
Figura 2

Comparação do número de ensaios clínicos registrados em relação ao perfil de morbimortalidade de crianças no Brasil.

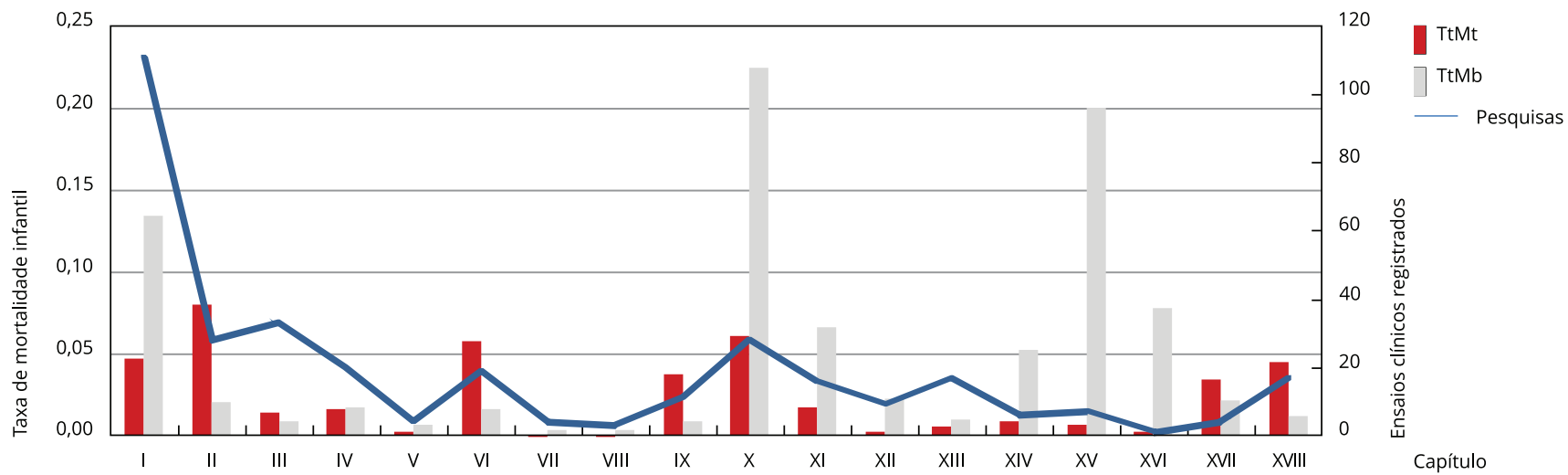

TtMb: proporção de hospitalizações registradas por Classificação Internacional de Doenças, 10a revisão (CID-10); TtMt: proporção de óbitos registrados por Classificação Internacional de Doenças.

Capítulos de CID-10 - I: doenças infecciosas e parasitárias; II: neoplasias; III: doenças do sangue e dos órgãos hematopoiéticos; IV: doenças endócrinas, nutricionais e metabólicas; V: transtornos mentais e comportamentais; VI: doenças do sistema nervoso; VII: doenças do olho e anexos; VIII: doenças do ouvido; IX: doenças do aparelho circulatório; X: doenças do aparelho respiratório;

XI: doenças do aparelho digestivo; XII: doenças da pele; XIII: doenças do sistema osteomuscular e do tecido conjuntivo; XIV: doenças do aparelho geniturinário; XV: gravidez, parto e puerpério; XVI: afecções originadas no período perinatal; XVII: malformações congênitas, deformidades e anomalias cromossômicas; XVIII: sintomas, sinais e achados anormais de exames clínicos e de laboratório.

Cabe destacar ainda que os ensaios clínicos de medicamentos para tratar doenças infecto-parasitárias contemplaram quase exclusivamente os antirretrovirais, provavelmente por estímulo da política brasileira de acesso ao tratamento de HIV/AIDS, tornando o país um grande mercado consumidor destes medicamentos 36 .

Um prognóstico de mercado levantado pela IMS Health - maior fornecedora de dados para prescrição médica nos Estados Unidos - indica que a perspectiva de consumo global de medicamentos estará focada principalmente em tratamentos nas áreas de infecção (14\%) e oncologia (13\%) até 202037. Essa tendência de consumo em nível internacional é acompanhada por um maior investimento de multinacionais nessas linhas de tratamento, conforme observado no presente trabalho.

A realização de ensaios clínicos constitui uma ferramenta importante para a geração de informações novas sobre os efeitos de tecnologia em saúde nos seres humanos 38. A efetivação de ensaios de qualidade pode prover respostas às mais variadas necessidades de saúde da população. A insuficiente relação observada entre os ensaios clínicos e a demanda de saúde do público pediátrico (Figura 2), parece decorrer da falta de capacitação e autonomia nacional para a realização destes ensaios, associada à dependência de multinacionais para a prática da pesquisa clínica 30 . Em 2005, a criação da chamada Rede Nacional de Pesquisa Clínica (RNPC) buscou fomentar estudos nacionais capazes de gerar respostas aos problemas de saúde da população brasileira 26. É possível, entretanto, que o desequilíbrio entre financiamento público e privado, demonstrado na Tabela 1, dificulte o fortalecimento da RNPC.

Por fim, cabe destacar a concentração de ensaios clínicos nos centros de pesquisa públicos e ligados a universidades federais e estaduais da Região Sudeste do país, já apontada por Zago 12, explicada, em parte, pela melhor infraestrutura e maior acesso à captação de recursos para a realização dos projetos, inclusive, de fontes públicas 39. A redução desse desequilíbrio regional e a utilização adequada da ampla rede de hospitais universitários, distribuídas nos outros estados do país, são dois aspectos relevantes, e ainda não superados, a serem estimulados no desenvolvimento da pesquisa clínica nacional 12 . 
As limitações relativas ao uso de dados secundários como fonte exclusiva de pesquisa, bem como o uso do recurso de busca avançada, no presente trabalho, devem ser consideradas. Pelo caráter voluntário dos registros (até o ano de 2012), a quantificação dos ensaios clínicos não permite, per si, a determinação de frequência e demais taxas, comumente verificadas em estudos epidemiológicos. Contudo, a caracterização obtida traz novos elementos para a discussão sobre a pesquisa, desenvolvimento e o uso de medicamentos em crianças e adolescentes. A estratégia de busca de ensaios clínicos incluindo a base ReBEC também foi adequada, visto que nove estudos estavam registrados somente no sistema brasileiro. Essa diferença era esperada, visto que tais ensaios clínicos eram coordenados por instituições nacionais que, possivelmente, não tinham interesse no registro internacional e focaram, exclusivamente, na recomendação brasileira (RDC no 39/2008) para a submissão posterior do registro do produto à Anvisa.

\section{Considerações finais}

O presente trabalho evidenciou a limitada prática dos ensaios clínicos pediátricos no Brasil, particularmente no que tange aos estudos conduzidos por iniciativa nacional.

O escasso incentivo governamental e a ampla dependência estrangeira parecem estar contribuindo para a realização de pesquisas clínicas pediátricas focadas nos interesses financeiros das empresas farmacêuticas. O país apresenta baixo esforço em pesquisa e pouco dinamismo no desenvolvimento de medicamentos em um mercado dominado por multinacionais há mais de 40 anos.

Ao que parece, será perdida mais uma onda tecnológica. Esperava-se que novos produtos, formas farmacêuticas, dispositivos para administrar medicamentos, testes laboratoriais e novas informações científicas mudassem a realidade do uso de medicamentos em crianças. É necessário definir uma política abrangente que envolva mudanças regulatórias, incentivo às indústrias, formação e financiamento de grupos de pesquisa clínica em pediatria e neonatologia, estímulo à farmácia pediátrica, para que o país, e não apenas alguns pacientes, em sua maioria do Sul e Sudeste, participem destes avanços.

Acredita-se que os resultados obtidos neste estudo quanto ao perfil dos ensaios clínicos envolvendo crianças brasileiras contribuirão para o debate no campo da saúde da criança e do adolescente.

\section{Colaboradores}

J. M. L. Vieira foi responsável por todos os aspectos do trabalho na garantia da exatidão e integridade de qualquer parte da obra. E. C. Lima contribuiu na concepção e projeto, análise e interpretação dos dados. M. G. P. Land, M. Ventura e H. L. L. Coelho colaboraram na redação do artigo e revisão crítica relevante do conteúdo intelectual. Todos os autores revisaram e provaram a versão final para publicação.

\section{Agradecimentos}

Ao Conselho Nacional de Desenvolvimento Científico e Tecnológico e Fundação de Amparo à Pesquisa do Estado do Rio de Janeiro.

\section{Referências}

1. Coelho HLL, Rey LC, Medeiros MSG, Barbosa RA, Fonseca SGC, Costa PQ. A critical comparison between the World Health Organization list of essential medicines for children and the Brazil list of essential medicines (Rename). J Pediatr (Rio J.) 2013; 89:171-8.

2. Burns JP. Research in children. Crit Care Med 2003; 31(3 Suppl):S131-6.

3. Costa PQ, Lima JES, Coelho HLL. Prescrição e preparo de medicamentos sem formulação adequada para crianças: um estudo de base hospitalar. Braz J Pharm Sci 2009; 45:57-66.

4. Liberato E, Souza PM, Silveira CAN, Lopes LC. Fármacos em crianças. In: Departamento de Assistência Farmacêutica e Insumos Estratégicos, Secretaria de Ciência, Tecnologia e Insumos Estratégicos, Ministério da Saúde, organizador. Formulário terapêutico nacional 2008: RENAME 2006. Brasília: Ministério da Saúde; 2008. p. 18-35. (Série B. Textos Básicos de Saúde). 
5. Fernandez E, Perez R, Hernandez A, Tejada P, Arteta M, Ramos JT. Factors and mechanisms for pharmacokinetic diferences between pediatric population and adults. Pharmaceutics 2011; 3:53-72.

6. Organização Pan-Americana da Saúde. Boas práticas clínicas: documentos das Américas. http://www.anvisa.gov.br/medicamentos/pes quisa/boasprati cas_americas.pdf (acessado em 21/Jun/2016).

7. Cadwell PH, Murphy SB, Butow PN, Craig JC. Clinical trials in children. Lancet 2004; 364:803-11.

8. Carvalho CG, Ribeiro MR, Bonilha MM, Fernandes Jr. M, Procianoy RS, Silveira RC. Uso de medicamentos off-label e não licenciados em unidade de tratamento intensivo neonatal e sua associação com escores de gravidade. J Pediatr (Rio J.) 2012; 88:465-70.

9. European Medicines Agency. 5-year Report to the European Commission. General report on the experience acquired as a result of the application of the Paediatric Regulation. London: European Medicines Agency; 2012.

10. Agência Nacional de Vigilância Sanitária. Resolução RDC no 39, de 5 de junho de 2008. Aprova o regulamento para a realização de pesquisa clínica e dá outras providências. Diário Ofical da União 2008; 6 jun.

11. Conselho Nacional de Saúde. Resolução no 466, de 12 de dezembro de 2012. Aprova diretrizes e normas regulamentadoras de pesquisas envolvendo seres humanos. Diário Oficial da União 2012; 13 jun.

12. Zago MA. A pesquisa clínica no Brasil. Ciênc Saúde Coletiva 2004; 9:363-74.

13. Dias AA, Galina SVR, Teixeira IG, Queiroz SR. Atividades de P\&D das multinacionais farmacêuticas no Brasil. Sistemas \& Gestão 2013; 8:458-68.

14. Cadwell PH, Murphy SB, Butow PN, Craig JC. Clinical trials in children. Lancet 2004; 364:803-11.

15. Gusson ACT, Lopes JC. Pediatria no século 21: uma especialidade em perigo. Rev Paul Pediatr 2010; 28:115-20.

16. International Committee of Medical Journal Editors. Clinical trial registration. http:// www.icmje.org/recommendations/browse/ publishing-and-editorial-issues/clinical-trialregistration.html (acessado em 24/Jun/2016).

17. Clinical trials: history, policies, and laws. https://clinicaltrials.gov/ct2/about-site/his tory (acessado em 24/Jun/2016).

18. The anatomical therapeutic chemical classification system with defined daily doses (ATC/ DDD). http://www.who.int/classifications/ atcddd/en/ (acessado em 26/Jun/2016).

19. Best pharmaceuticals for children act. https:// bpca.nichd.nih.gov/Pages/Index.aspx (acessado em 27/Jun/2016).

20. Hoppu K, Anabwani G, Garcia-Bournissen F, Gazarian M, Kearns GL, Nakamura H, et al. The status of paediatric medicines initiatives around the world - what has happened and what has not? Eur J Clin Pharmacol 2012; 68:1-10.
21. Vickers AJ. Clinical trials in crisis: four simple methodologic fixes. Clin Trials 2014; 11:615-21.

22. Tsoulfas G. The impact of the European financial crisis on clinical research within the European Union or when life gives you lemons, make lemonade. Hippokratia 2012; 16:6-10.

23. Pammolli F, Magazzini L, Riccaboni M. "The productivity crisis in pharmaceutical R\&D”. Nat Rev Drug Discov 2011; 10:428-38.

24. Moses 3rd H, Matheson DH, Cairns-Smith S, George BP, Palisch C, Dorsey ER. The anatomy of medical research US and international comparisons. JAMA 2015; 313:174-89.

25. Gomes RP, Pimentel VP, Landim AB, Pieroni JP. Ensaios clínicos no Brasil: competitividade internacional e desafios. BNDES Setorial 2012; 36:45-84.

26. Ministério da Saúde. Rede Nacional de Pesquisa Clínica do Brasil: respostas e redução da dependência estrangeira. Rev Saúde Pública 2010; 44:575-8

27. Karlberg JPE, Speers MA. Revisão de estudos clínicos: um guia para o comitê de ética. Hong Kong: Karlberg, Johan Petter Einar; 2010.

28. Goldim JR. A avaliação ética da investigação científica de novas drogas: a importância da caracterização adequada das fases da pesquisa. Rev HCPA \& Fac Med Univ Fed Rio Gd do Sul 2007; 27:66-73.

29. Diniz D, Guilhem D. O que é bioética. São Paulo: Editora Brasiliense; 2002.

30. Quental C, Salles Filho S. Ensaios clínicos: capacitação nacional para avaliação de medicamentos e vacinas. Rev Bras Epidemiol 2006; 9:408-24.

31. ZoboliE,Oselka G. Conflito de interesses napesquisa clínica. Revista Bioética 2007; 15:65-76.

32. Duarte D, Fonseca H. Melhores medicamentos em pediatria. Acta Pediatr Port 2008; 39:17-22.

33. van Riet-Nales DA, De Neef BJ, Schobben AF, Ferreira JA, Egberts TC, Rademaker CM. Acceptability of different oral formulations in infants and preschool children. Arch Dis Child 2013; 98:725-31.

34. Nunn T, Williams J. Formulation of medicines for children. Br J Clin Pharmacol 2005; 59:674-6.

35. Souza Junior AS, Santos DB, Rey LC, Medeiros MG, Vieira MG, Coelho HL. Off-label use and harmful potential of drugs in a NICU in Brazil: a descriptive study. BMC Pediatr 2016; 16:13.

36. Vidotti CCF, Castro LLC. Fármacos novos e necessidades do Sistema Único de Saúde no Brasil. Revista Espaço para a Saúde 2009; 10:7-11.

37. IMS Health. Global medicines use in 2020: outlook and implications. http://www.im shealth.com/en/thought-leadership/imsinstitute/reports/global-medicines-use-in2020\#ims-form (acessado em 28/Jun/2016).

38. Ministério da Saúde. Avaliação de tecnologias em saúde. Ferramentas para a gestão do SUS. Brasília: Editora Ministério da Saúde; 2009. (Série A. Normas e Manuais Técnicos).

39. Ministério da Saúde. Fortalecendo à pesquisa clínica no Brasil: a importância de registrar os ensaios clínicos. Rev Saúde Pública 2011; 45:436-9. 


\section{Abstract}

This study aimed to characterize the clinical trials with medicines enrolling Brazilian children and adolescents, registered in the databases of Clinical Trials and the Brazilian Clinical Trials Network (ReBEC) from 1994 to 2014. Only 462 clinical trials enrolled Brazilian children and adolescents. There was an increase in registrations beginning in 2003, with an important drop in 2011. Among these trials, 35.5\% were hosted in Brazil. The international clinical trials were mostly conducted by North American companies. In both cases, multinational industry was the principal source of funding. The clinical trials were predominantly phase III with injectable and solid oral pharmaceutical forms of antiviral drugs. Domestic clinical trials showed wider variation in the pharmaceutical forms and higher percentage of liquid formulations, when compared to the international trials. In addition to heavy external dependence for conducting clinical trials, the study emphasized the challenge for pediatric care in Brazil, which presents epidemiological peculiarities in an environment prone to the use of unlicensed medicines for children.

Clinical Trial; Pediatrics; Health Information Systems

\section{Resumen}

Este estudio tuvo como objetivo caracterizar los ensayos clínicos con medicamentos, involucrando a niños y adolescentes brasileños, registrados en las bases de datos de Clinical Trials y de la Red Brasileña de Ensayos Clínicos (ReBEC), entre los años de 1994 y 2014. Solamente 462 ensayos clínicos involucraron a brasileños en esa franja de edad. A partir de 2003, hubo un aumento en el número de registros, con una expresiva caída en 2011. Entre ellos, un 35,5\% estuvieron ubicados en Brasil. Los ensayos clinicos internacionales fueron mayoritariamente dirigidos por empresas norteamericanas. En ambos casos, la industria multinacional fue la principal fuente de apoyo financiero. Predominaron ensayos clínicos de fase III con antivirales en formas farmacéuticas inyectables $y$ orales sólidas. Los ensayos clínicos nacionales presentaron una mayor variación, en cuanto a las formas farmacéuticas y mayor porcentaje de formulaciones líquidas investigadas, en comparación con los internacionales. Además de la fuerte dependencia externa para la realización de los ensayos clínicos, se destacó el desafío para el cuidado pediátrico en Brasil, que presenta peculiaridades epidemiológicas en un ambiente propicio al uso de medicamentos sin licencia para niños.

Ensayo Clínico; Pediatría; Sistemas de Información en Salud
Recebido em 14/Out/2015

Versão final reapresentada em 30/Jun/2016

Aprovado em 13/Jul/2016 